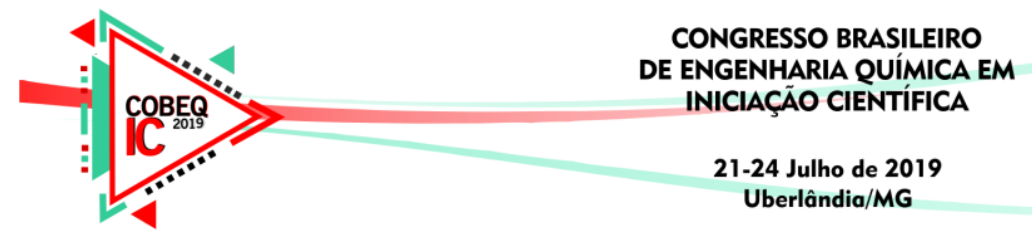

\title{
AVALIAÇÃO EXPERIMENTAL DO COEFICIENTE EXTERNO DE CONVECÇÃO NO RESFRIAMENTO DE LÍQUIDOS NEWTONIANOS EM TANQUE COM AGITAÇÃO
}

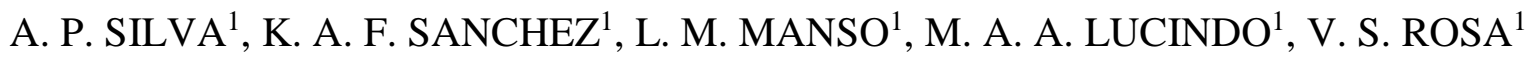 \\ ${ }^{1}$ Universidade Santa Cecília, Faculdade de Engenharia Química \\ E-mail para contato: arianepazzini@ hotmail.com
}

\begin{abstract}
RESUMO - Os impulsores tipo turbina Rushton são amplamente empregados em processos de transferência de calor e massa em tanques com agitação. A determinação do coeficiente externo de convecção é imprescindível para o cálculo da área de troca térmica de jaquetas, serpentinas e chicanas. O presente estudo visou avaliar a variação temporal do coeficiente ho no resfriamento de líquidos newtonianos em tanque equipado com uma turbina Rushton e chicanas tubulares verticais. Os experimentos foram conduzidos em uma unidade experimental equipada com um tanque com volume de 10 litros, uma turbina Rushton e uma chicana tubular vertical. Utilizou-se líquidos newtonianos durante a agitação e resfriamento. Com os resultados obtidos, verificou-se que o coeficiente externo de convecção no resfriamento possui uma variação temporal devido aos elevados gradientes de temperatura. O modelo empírico proposto para o cálculo do coeficiente de convecção é válido para Reynolds entre 2000 e 50000, com um coeficiente de determinação de 0,78 .
\end{abstract}

\section{INTRODUÇÃO}

Os tanques com impulsores mecânicos são amplamente utilizados nas indústrias químicas, petroquímicas, farmacêuticas e alimentícias como reatores, decantadores, extratores e destiladores (Rosa, et. al., 2014). O tipo de impulsor mecânico (axial ou radial) irá impactar diretamente no consumo de potência e na transmissão de calor pois o tipo de escoamento altera drasticamente a hidrodinâmica da agitação e, por consequência, a transferência de calor do líquido na mistura (Ameur e Bouzit, 2013). Industrialmente, as turbinas Rushton (impulsor do tipo radial com 6 pás planas) são bastante atrativas para processos em que uma elevada turbulência é requerida, como por exemplo, operações de transferência de calor e massa, flotação e reações químicas entre gás e líquido (Gómez-Días e Navaza, 2004). A área de troca térmica, independente do tipo de trocador de calor utilizado no tanque, é determinada em função dos mecanismos de transmissão de calor. No entanto, a resistência a transferência de calor ocasionada pela condução e radiação são desprezíveis frente a convecção que é função do escoamento (Dhotre, Murthy e Jayakumar, 2006). Desse modo, a área de troca térmica é calculada como função do coeficiente global de transferência de calor (U), o qual é função dos coeficientes de convecção interno $\left(\mathrm{h}_{\mathrm{i}}\right)$ e externo $\left(\mathrm{h}_{\mathrm{o}}\right)$ como apresentado na Equação 1.

$$
\frac{1}{\mathrm{U}}=\frac{1}{\mathrm{~h}_{\mathrm{i}}}+\frac{1}{\mathrm{~h}_{\mathrm{o}}}
$$




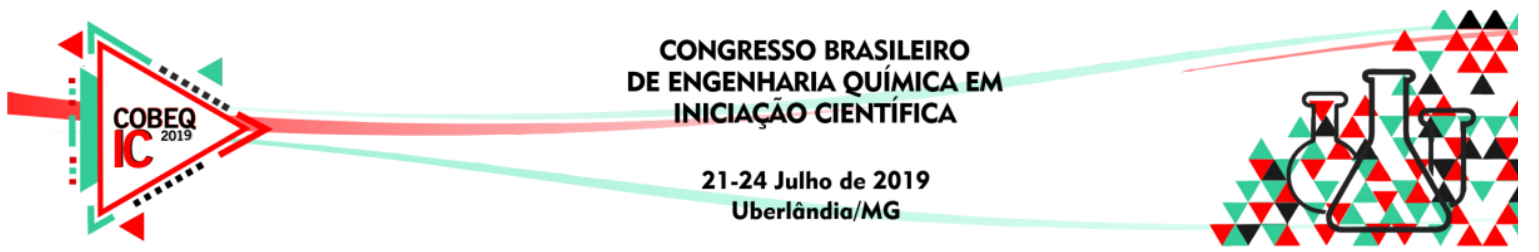

A dificuldade de cálculo na Equação 2 envolve a determinação do coeficiente externo de convecção $\left(h_{0}\right)$ o qual depende de parâmetros como a geometria do tanque e do impulsor mecânico, das propriedades físicas do líquido e da reologia do líquido. Tradicionalmente, o coeficiente $h_{o}$ é determinado pelo modelo semi-empírico de Nusselt (Nu) (Equação 2) em função do número de Reynolds (Re), número de Prandtl (Pr) e número de Viscosidade (Vi).

$$
\mathrm{Nu}=\mathrm{KRe}^{\mathrm{a}} \operatorname{Pr}^{\mathrm{b}} \mathrm{Vi}^{\mathrm{c}}
$$

$\mathrm{O}$ uso da Equação 1 requer que o coeficiente $\mathrm{h}_{\mathrm{o}}$ presente no número de Nusselt seja constante para uma dada rotação do impulsor mecânico independente se o processo esteja em regime transiente e permanente. Porém, no trabalho de Rosa et. al. (2017) com aquecimento de líquidos newtonianos em tanques utilizando serpentinas em espiral, verificou-se que há uma tendência do coeficiente $h_{o}$ não ser completamente constante em função do tempo.

\section{OBJETIVO}

O presente estudo teve por objetivo avaliar a variação temporal do coeficiente $h_{0}$ no resfriamento de líquidos newtonianos em tanque equipado com uma turbina Rushton e chicanas tubulares verticais. O estudo também contemplou determinar um modelo empírico para o cálculo do coeficiente $h_{\mathrm{o}}$ em função do escoamento.

\section{MATERIAL E MÉTODO}

\subsection{Material}

Os experimentos foram conduzidos em uma unidade experimental, localizada no Laboratório de Operações Unitárias da Universidade Santa Cecilia. Na Figura 1 está apresenta um fluxograma da unidade com o detalhamento dos seus principais periféricos.

Figura 1 - Fluxograma da unidade Experimental -- (1) Banho termostático; (2) Bomba; (3) Válvulas de esfera; (4)Termopar 1 (entrada do fluido); (5) Chicana tubular vertical, (6) Termopar temperatura bulk 1 (tanque); (7) Isolamento térmico; (8) Tanque; (9) Termopar temperatura bulk 2; (10) Termopar temperatura bulk 3; (11) Impulsor mecânico; (12) Motor elétrico; (13) Termopar 2 (saída do fluido); (14) válvula de esfera; (15) Rotâmetro;

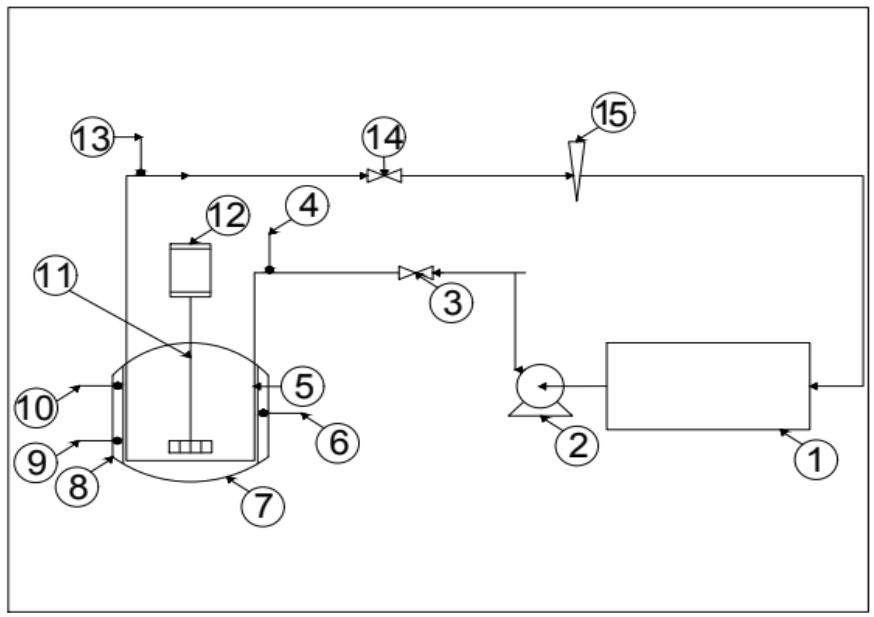




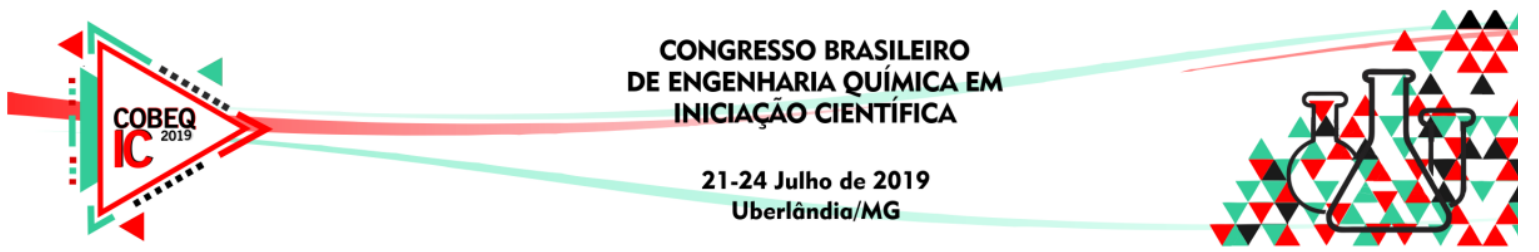

Nas Figuras 2 e 3 estão apresentados a chicana tubular vertical e a turbina Rushton com 6 pás planas, respectivamente. Como fluido a serem resfriados foram empregados: água destilada, soluções de sacarose com concentrações em massa de $20 \%$ e $50 \%$ e os mesmos foram empregados na agitação. Como fluido de resfriamento, utilizou-se água destilada que percorreu em sistema fechado um circuito entre a chicana tubular vertical e o banho ultratermostático.

Figura 2 - Chicana tubular vertical de cobre

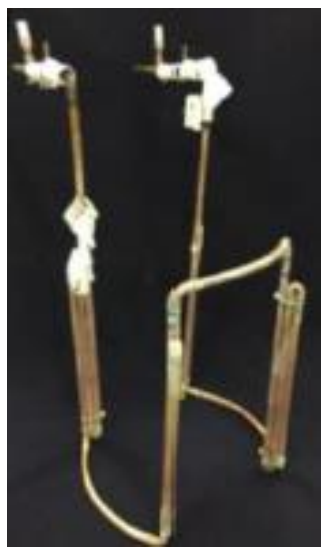

Figura 3 - Turbina Rushton com 6 pá planas

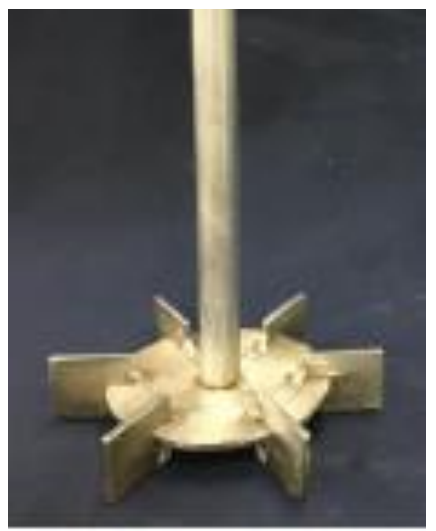

\subsection{Método}

O método empregado nesse estudo foi uma operação em batelada e de resfriamento de líquidos em agitação e mistura. Cada líquido (água e soluções de sacarose a $20 \%$ e $50 \%$, em massa), foram submetidas a diferentes níveis de rotações da turbina na faixa de $100 \mathrm{rpm}$ a 700 rpm. O líquido no tanque (volume de 10 litros) era aquecido previamente até $50^{\circ} \mathrm{C}$ por meio de uma resistência elétrica, enquanto, que a água de resfriamento era mantida em recirculação no banho ultratermostático a $15^{\circ} \mathrm{C}$ com uma vazão de 1,5 litros por minuto. Após as temperaturas de ambos os líquidos estarem nas condições de estudo, a água de resfriamento era direcionada a chicana tubular vertical, onde o resfriamento do líquido em agitação era iniciado. Foram acoplados termopares na entrada e na saída da chicana tubular vertical, bem como foram colocados 3 termopares em posições distintas no interior do tanque. Cada ensaio teve a duração de 40 minutos, de modo, que a cada 1 minuto eram medidas as temperaturas em todos os termopares supracitados. O tanque era isolado com espuma de poliuretano. A sequência de cálculo está na determinação do coeficiente global de transferência de calor (U), coeficiente interno de convecção $\left(h_{i}\right)$ e coeficiente externo de convecção $\left(h_{o}\right)$. Inicialmente aplicou-se a $1^{\text {a }}$ lei da termodinâmica no volume de controle (tanque com resfriamento), conforme apresentado na Equação 3.

$$
\sum \dot{\mathrm{Q}}-\sum \dot{\mathrm{W}}+\sum \dot{\mathrm{m}}_{\mathrm{e}}\left(\mathrm{h}_{\mathrm{e}}+\frac{\mathrm{ve}_{\mathrm{e}}^{2}}{2}+\mathrm{gZ}_{\mathrm{e}}\right)-\sum \dot{\mathrm{m}}_{\mathrm{s}}\left(\mathrm{h}_{\mathrm{s}}+\frac{\mathrm{v}_{\mathrm{s}}^{2}}{2}+\mathrm{gZ}_{\mathrm{s}}\right)=\frac{\mathrm{dE}}{\mathrm{d} \theta}
$$

Considerando as seguintes premissas: a) tanque perfeitamente isolado, de modo, que o calor removido do líquido em agitação era completamente transferido para a água de resfriamento na chicana tubular vertical, b) Energia cinética e energia potencial desprezíveis, c) Trabalho do impulsor mecânico desprezível e d) tanque perfeitamente agitado, a Equação 3 com as devidas manipulações algébricas se reduz a: 


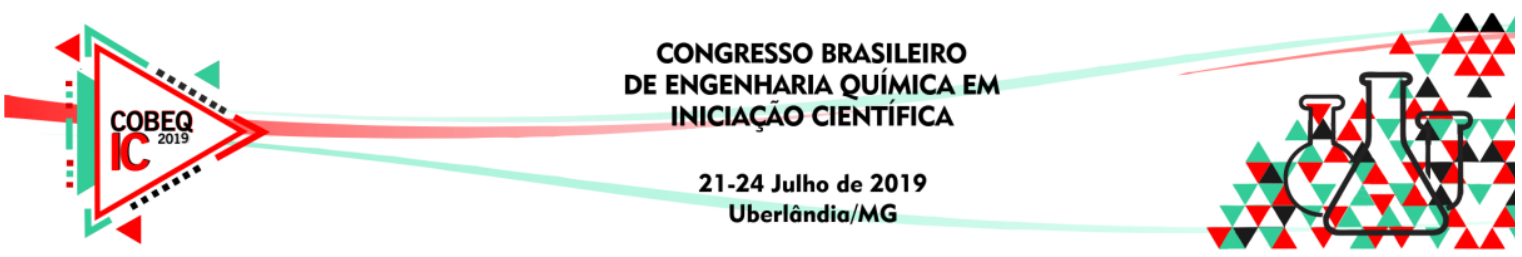

$$
U=\frac{1}{A\left(T_{1}-T_{2}\right)} \cdot \ln \left(\frac{T_{1}-t_{b}}{T_{2}-t_{b}}\right) \cdot\left(M c_{p} \frac{d t_{b}}{d \theta}\right)
$$

A Equação 4 não pode ser integrada analiticamente, pois ainda não é possível conhecer se o coeficiente U é uma função do tempo. Desse modo, foi realizada uma discretização na Equação 4, conforme apresentado na Equação 5, e a integração realizada de modo numérico.

$$
U^{k}=\frac{1}{A^{k}\left(T_{1}{ }^{k}-T_{2}{ }^{k}\right)} \cdot \ln \left(\frac{T_{1}{ }^{k}-t_{b}{ }^{k}}{{T_{2}}^{k}-t_{b}{ }^{k}}\right) \cdot\left\{\left[M c_{p}{ }^{k}\left(\frac{t_{b}{ }^{k}-t_{b}{ }^{k-1}}{\theta^{k}-\theta^{k-1}}\right)\right]\right\}
$$

O coeficiente hi foi calculado pelo Equação de Gnielinski (1976) a qual é válida para escoamento interno em tubulações (chicana tubular vertical) com Reynolds entre $3.10^{3}$ e $5.10^{6}$. Por fim, o coeficiente ho, é calculado através da Equação 1 com os resultados encontrados nas Equações 4 e 5 para cada experimento.

$$
\frac{h_{i} D_{i}}{k}=\frac{\left(f^{\prime} / 8\right)(R e-1000) P r}{1+12,7\left(f^{\prime} / 8\right)^{1 / 2}\left(\operatorname{Pr}^{2 / 3}-1\right)}
$$

\section{RESULTADOS E DISCUSSÃO}

Inicialmente, verificou-se a influência da agitação e o tempo de operação no coeficiente interno de convecção $\left(h_{i}\right)$. Em todos os ensaios, a água de resfriamento foi conduzida a 1,5 litros por minuto, e por meio da Equação 6, aplicada a cada instante de tempo, verificou-se que o coeficiente $h_{i}$ permaneceu constante em cada experimento com um valor de $3600 \mathrm{~W} / \mathrm{m}^{2 \circ} \mathrm{C}$. Desse modo, calculou-se o coeficiente U em cada instante de tempo, através da Equação 5, e por sua vez, o coeficiente $h_{\mathrm{o}}$ foi obtido pela Equação 1. Na Figura 4 está apresentada a curva de variação do coeficiente $h_{o}$ em função do tempo para o ensaio de resfriamento da solução de sacarose a $50 \%$ em massa com uma rotação da turbina em 300 rpm. Observou-se na Figura 4 que houve uma significativa variação do coeficiente $h_{0}$ com o tempo de resfriamento. O mesmo ocorreu para todos os outros experimentos.

Fenomenologicamente, a rampa de resfriamento é mais brusca quando comparada com a rampa de aquecimento. No início dos experimentos, a transferência de calor é extremamente acentuada, o que provoca gradientes de temperatura significativos no líquido em agitação. Devido a esses gradientes, as propriedades físicas, em especial a viscosidade, sofre uma mudança brusca, o que reflete na mudança do coeficiente $h_{o}$ com o tempo. Constatou-se que há um amortecimento da variação do coeficiente $h_{o}$ a partir de 25 minutos de experimento, de forma que a função pode ser ajustada exponencialmente (Equação 7).

$$
h_{o}=k_{1} e^{k_{2} \theta}
$$

No entanto, a Equação 7 não é funcional, pois a mesma só pode ser aplicada a uma condição específica de experimento. As constantes $\mathrm{k}_{1}$ e $\mathrm{k}_{2}$ devem ser escritas em função do número de Reynolds, de modo, a expandir a aplicação do modelo empírico para o cálculo do coeficiente $h_{0}$. 


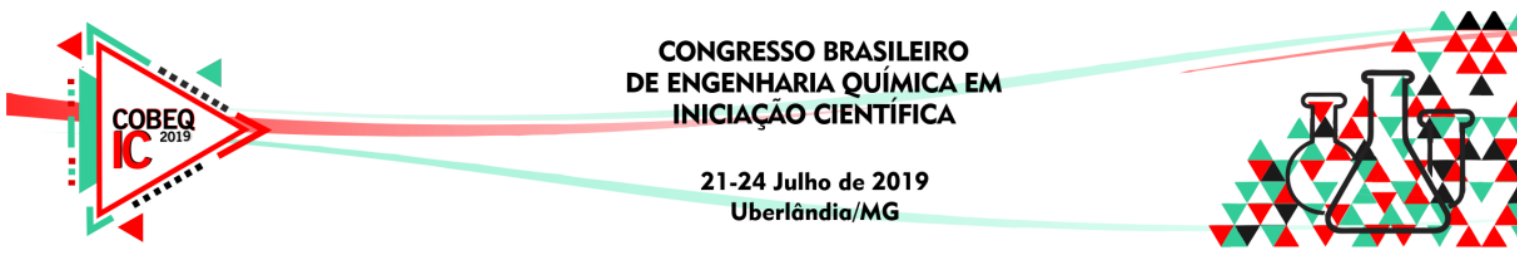

Figura 4 - Coeficiente $h_{0}$ em função do tempo para resfriamento de solução de sacarose a 50\% com rotação da turbina em $300 \mathrm{rpm}$

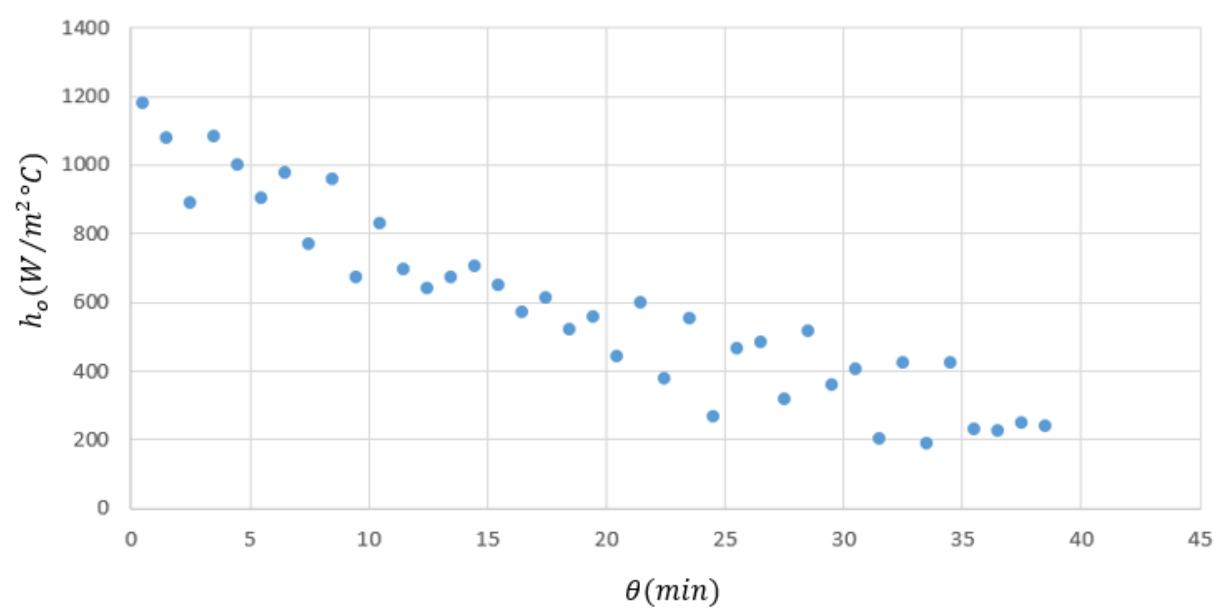

Na Figura 5a foi plotado a variação do coeficiente $\mathrm{k}_{1}$ em função do número de Reynolds (calculado a partir de cada rotação empregada) e na Figura 5b, a relação do coeficiente $\mathrm{k}_{2}$ pelo número de Reynolds. Ambos os coeficientes, $\mathrm{k}_{1}$ e k2, possuíram uma variação similar com o número de Reynolds, sendo, que o ajuste entre os parâmetros é melhor ajustado com uma função logarítmica. Desse modo, a equação 7 pode ser reescrita em função do número de Reynolds, como apresentado na Equação 8, a qual é aplicável para Reynolds entre 2000 e 50000.

$$
h_{o}=[695,79 \ln R e-3170,1] \cdot \exp ^{(0,0117-0,008 \ln R e) \theta}
$$

Figura 5 - (a) Coeficiente $\mathrm{k}_{1}$ em função de Reynolds, (b) Coeficiente $\mathrm{k}_{2}$ em função de Reynolds

(a)

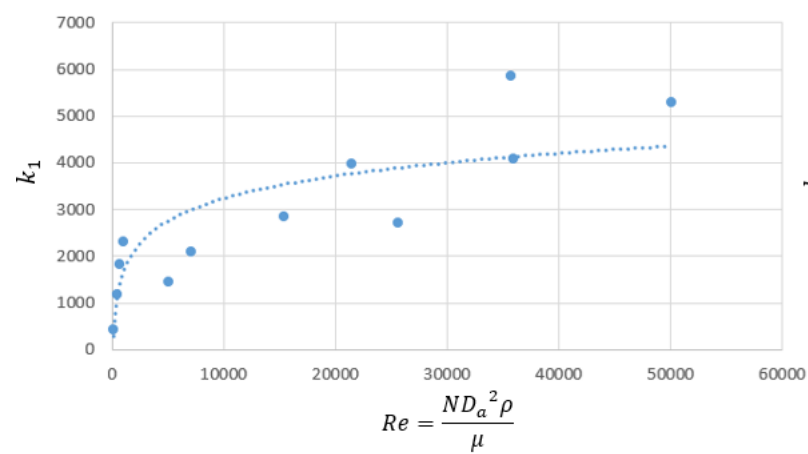

(b)

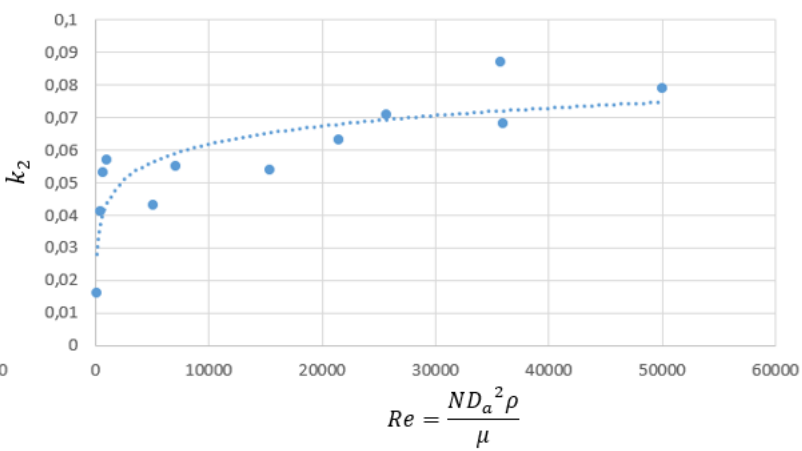

\section{CONCLUSÃO}

Com os resultados obtidos, foi possível concluir que: a) No resfriamento de líquidos newtonianos, devido ao elevado gradiente de temperatura, a variação das propriedades físicas provoca uma mudança do coeficiente externo de convecção com o tempo; b) O modelo empírico obtido para o coeficiente $\mathrm{h}_{\mathrm{o}}$ segue um modelo exponencial com os seus coeficientes ajustados por funções logarítmicas com o número de Reynolds. A equação é válida para resfriamentos com turbinas Rushton com Reynolds com intervalo de 2000 a 50000 com um coeficiente $\mathrm{R}^{2}$ de 0,78 . 


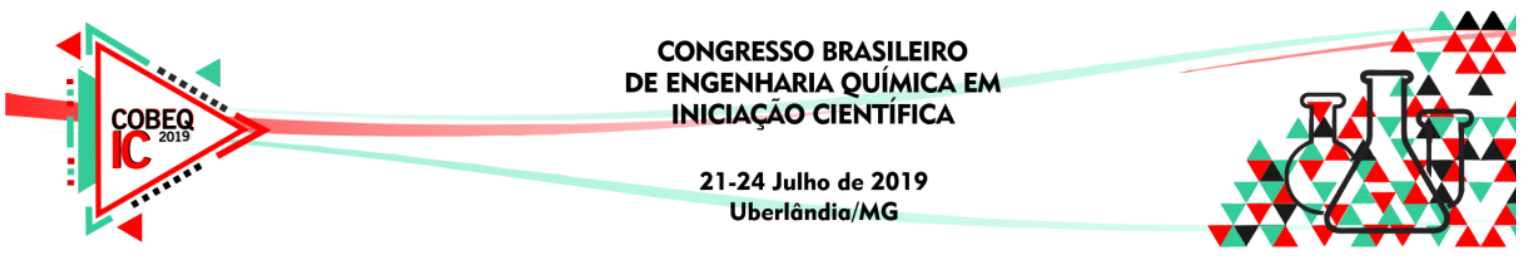

\section{NOMENCLATURA}

$\mathrm{U}$

$$
\begin{gathered}
\text { Coeficiente } \\
\text { global de } \\
\text { transferência de } \\
\text { calor }\left(\mathrm{W} / \mathrm{m}^{2 \circ} \mathrm{C}\right)
\end{gathered}
$$

A

$$
\begin{gathered}
\text { Área de troca } \\
\text { térmica }\left(\mathrm{m}^{2}\right)
\end{gathered}
$$

Temperatura de
entrada na
chicana tubular $\left({ }^{\circ} \mathrm{C}\right)$

$$
\begin{gathered}
\text { Temperatura de } \\
\text { saída na chicana } \\
\text { tubular }\left({ }^{\circ} \mathrm{C}\right)
\end{gathered}
$$

tb

$\operatorname{Pr}$

$$
\text { Temperatura }
$$
"bulk" no tanque $\mathrm{k}$ integração na equação 5

$\mathrm{cp}$

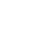

$$
\text { Número de }
$$
Prandtl

\section{ho

(1) \\ Coeficiente externo de convecção $\left(\mathrm{W} / \mathrm{m}^{2 \circ} \mathrm{C}\right)$}

f

Da

Diâmetro do

$$
\begin{gathered}
\text { Calor } \\
\text { específico } \\
(\mathrm{J} / \mathrm{kgK})
\end{gathered}
$$

Coeficiente
interno de
convecção
$\left(\mathrm{W} / \mathrm{m}^{2}{ }^{\circ} \mathrm{C}\right)$

Fator de atrito impulsor (m)
Re $\quad \begin{gathered}\text { Número de } \\ \text { Reynolds }\end{gathered}$
a

Q

Fluxo de calor no volume de controle

(W)

$\mathrm{Nu} \quad$ Número de
Nusselt

W

Trabalho no volume de controle

(W)

\begin{tabular}{cccc}
\hline & $\begin{array}{c}\text { Viscosidade } \\
\text { dinâmica (Pa.s) }\end{array}$ & E & $\begin{array}{c}\text { Energia no } \\
\text { volume de } \\
\text { controle }(\mathrm{J})\end{array}$ \\
$\rho \quad$ & $\begin{array}{c}\text { Massa } \\
\text { específica } \\
\left(\mathrm{kg} / \mathrm{m}^{3}\right)\end{array}$ & $\theta$ & $\begin{array}{c}\text { Tempo do } \\
\text { ensaio } \\
(\mathrm{min})\end{array}$
\end{tabular}

$\mathrm{N}$

Rotação (rpm)

M

Massa de líquido (kg)

\section{REFERÊNCIAS}

ROSA, V. S., MORAES, M. S., TONELI, J. T. C. L., MORAES JÚNIOR, D. "External heat transfer coefficient in agitated vessels using a radial impeller and vertical tube baffles". Industrial \& Engineering Chemistry Research, 53 (35), p. 13797-13803, 2014;

AMEUR, H., BOUZIT, M. "Power consumption for stirring shear-thinning fluids by two-blade impeller”. Energy, 50, p.326-332, 2013;

GÓMEZ-DÍAS, D., NAVAZA, J. M., “Analysis of carbon dioxide gas/liquid mass transfer in aerated stirred vessels using non-newtonian media". Journal of Chemical Technology and Biotechnology, 79, p. 1105-1112, 2004;

DHOTRE, M. T., MURTHY, Z. V. P., JAYAKUMAR, N. S., "Modeling \& Dynamic Studies of Heat Transfer Cooling of Liquid in Half-coil Jackets". Chemical Engineering Journal, 118, p. 183-188, 2006;

ROSA, V.S., TAQUEDA, M. E. S., PAIVA, J. L., MORAES, M. S., MORAES JÚNIOR, D. "Nusselt's correlations in agitated tanks using the spiral coil with Rushton turbine and PBT $45^{\circ}$ impeller. Comparison with tanks containing vertical tube baffles". Applied Thermal Engineering, 110, p. 1331-1342, 2017; 